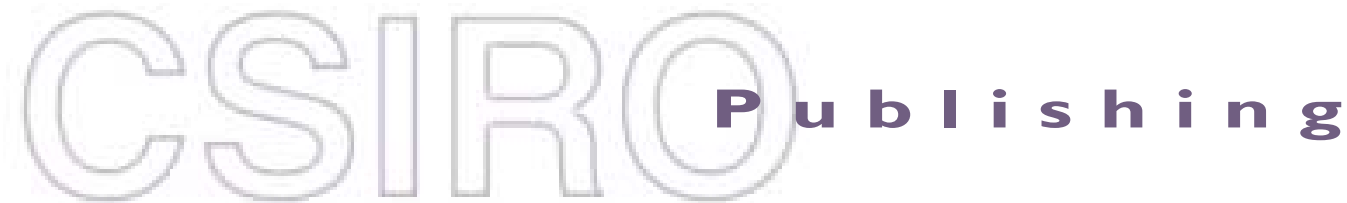

\section{Publications of the Astronomical Society of Australia}

Volume 19, 2002

(C) Astronomical Society of Australia 2002

An international journal of astronomy and astrophysics

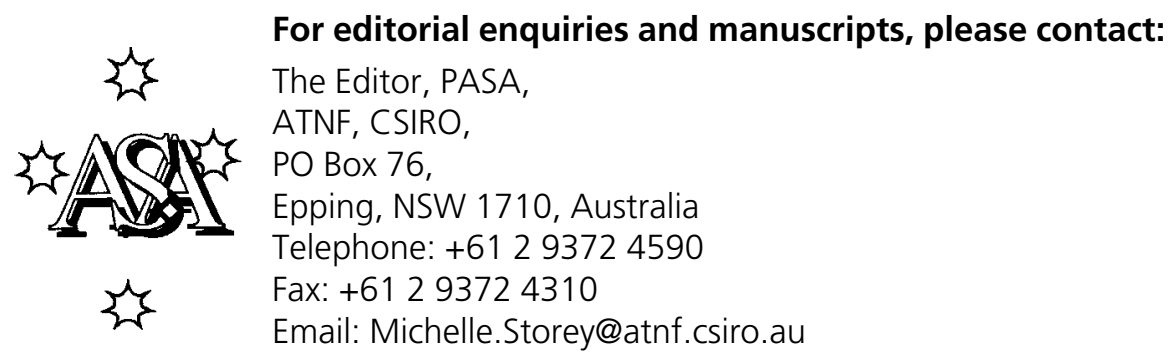

For general enquiries and subscriptions, please contact: CSIRO Publishing PO Box 1139 (150 Oxford St)

Collingwood, Vic. 3066, Australia

Telephone: +6139662 7666

Fax: +61 396627555

Email: publishing.pasa@csiro.au

C S I RO

PUBLISHING Published by CSIRO Publishing

for the Astronomical Society of Australia

www.publish.csiro.au/journals/pasa 


\title{
New Results from an ATCA Study of Intraday Variable Radio Sources
}

\author{
H. E. Bignall ${ }^{1,2}$, D. L. Jauncey ${ }^{2}$, L. L. Kedziora-Chudczer ${ }^{2,3}$, J. E. J. Lovell ${ }^{2}$, \\ J.-P. Macquart ${ }^{4,5}$, D. P. Rayner ${ }^{2}$, S. J. Tingay ${ }^{2}$, A. K. Tzioumis ${ }^{2}$, \\ R. W. Clay ${ }^{1}$, R. G. Dodson ${ }^{6}$, P. M. McCulloch ${ }^{6}$ and G. D. Nicolson ${ }^{7}$ \\ ${ }^{1}$ Department of Physics, University of Adelaide, SA 5005, Australia \\ hbignall@physics.adelaide.edu.au \\ ${ }^{2}$ Australia Telescope National Facility, CSIRO, Epping, NSW, Australia \\ ${ }^{3}$ Anglo-Australian Observatory, Epping, NSW, Australia \\ ${ }^{4}$ Research Centre for Theoretical Astrophysics, School of Physics, \\ University of Sydney, NSW 2006, Australia \\ ${ }^{5}$ now at Kapteyn Institute, University of Groningen, The Netherlands \\ ${ }^{6}$ School of Maths and Physics, University of Tasmania, Hobart, Tasmania 7001, Australia \\ ${ }^{7}$ Hartebeesthoek Radio Astronomy Observatory, Krugersdorp 1740, Transval, South Africa \\ Received 2001 September 4, accepted 2001 November 6
}

\begin{abstract}
We are undertaking an observational program using the ATCA to monitor the intraday variability (IDV) of a sample of sources at 4.8 and $8.6 \mathrm{GHz}$. The sources were selected to include the known strong southern IDV sources plus a number of sources whose IDV was recently discovered. The present monitoring program will extend over a full year in order to search for any annual cycle that may be present in the longterm IDV characteristics of these sources. In this paper we discuss the observing strategy and data analysis, and present the first results from our observations.
\end{abstract}

Keywords: radio continuum: galaxies — quasars: individual (PKS 1257-326, PMN J1326-5256, PMN J1047-6217) — ISM: general — polarisation

\section{Introduction}

Intraday variability (IDV) observed in extragalactic sources at $\mathrm{cm}$ wavelengths implies sources small enough to be affected by interstellar scintillation (ISS). The characteristic timescale, $t_{\text {char }}$, of variability due to ISS is inversely proportional to the velocity of the scintillation pattern, $\mathbf{v}_{\text {ISS }}$, which is moving past the observer. A change in $t_{\text {char }}$ will be observable if there is a substantial change in the relative transverse velocity of the source, screen, and observer. If at some time during the year, the Earth's orbital velocity is parallel to the velocity of the ISM in the plane perpendicular to the line-of-sight to the source, then the relative velocity is small and so $t_{\text {char }}$ is long. At other times, when the Earth's velocity is anti-parallel to the ISM velocity, the relative velocity is large and $t_{\text {char }}$ is correspondingly short.

The detection of an annual modulation in $t_{\text {char }}$ for $\mathrm{J} 1819+3845$ has established ISS as the principal mechanism responsible for $\mathrm{cm}$ wavelength IDV in this source (Dennett-Thorpe \& de Bruyn 2001). An annual modulation has also been suggested for the long-lived IDV source 0917+624 (Jauncey \& Macquart 2001; Rickett et al. 2001), based on previously published data (Heeschen et al. 1987; Kraus et al. 1999). Two observations 13 years apart, which showed $t_{\text {char }}$ in this source to be much longer than at other times, were the only lengthy observations of the source during the expected slow-down period before 2000 , prompting further observations to confirm an annual modulation. However Fuhrmann et al. (2001) report that, after slowing down at about the expected epoch in September 2000, 0917+624 has not returned to its 'normal' fast IDV behaviour, casting doubt on the existence of an annual period in this source. Alternatively, this prolonged slowing of the variations could be due to a change in the mean properties of the ISM in the line-of-sight or the intrinsic source structure, which could disrupt an annual cycle. Further observations are required to resolve this question.

The observed annual cycle in $\mathrm{J} 1819+3845$ indicates that, for the line of sight towards this source, the ISM speed and the Earth's speed are comparable. For pulsars, high proper motions often dominate $\mathbf{v}_{\text {ISS }}$, so it is generally not possible to observe an annual cycle in the scintillation timescale. Source structure, and/or anisotropy in ISM turbulence, can also introduce a variation in $t_{\text {char }}$, as the direction of $\mathbf{v}_{\text {ISS }}$ changes over the course of a year.

In this paper we describe a new observational program, started in February 2001, to look for annual cycles in a sample of sources in which IDV has previously been detected. If an annual cycle is found, this will confirm that the IDV is predominantly due to interstellar scintillation. From the annual cycle in $t_{\text {char }}$, it is possible to derive the velocity of the scattering screen, and information on source and ISM structure. This technique probes structure on scales comparable with the angular scale of the scintillation pattern, of order $\sim 10 \mu$ as, thus achieving higher angular resolution than is currently available with space VLBI. Observations of IDV behaviour in polarised as well as total flux density may help to model source structure, 
as often IDV behaviour in polarised flux density differs from the IDV in total flux density, indicating polarised substructure in the source.

\section{The Source Sample}

The sample, listed in Table 1, is made up of flat-spectrum sources (Heeschen 1984) that have shown strong IDV in the ATCA IDV Survey (Kedziora-Chudczer et al. 2001), or have been found in other IDV surveys (Quirrenbach et al. 1992; Romero, Combi, \& Colomb 1994). In addition we have added several sources whose IDV we have recently discovered. The ATCA Survey found that sources which exhibited strong variability on a month-to-month timescale were also likely IDV sources. In this way, we have since found strong IDV in PMN J1047-6217, PMN J1326-5256, and PKS 1622-253. PKS 1257-326 was found serendipitously in the Deep X-ray Radio Blazar Survey (Perlman et al. 1998), and PKS 1415-349 showed IDV in an ATCA Gravitational Lens Survey (Lovell 1997; J. E. J. Lovell et al., in preparation). We also include several compact, non-IDV sources for calibration of changes in gain due to atmospheric and antenna-based effects.

\section{The Observations}

Our observing strategy is to observe with the ATCA for 48 contiguous hours, approximately every $6-8$ weeks over the course of a year, to follow variations in $t_{\text {char }}$. When possible, these data are supplemented with single-dish flux density measurements obtained with the University of Tasmania's $30 \mathrm{~m}$ antenna at Ceduna and the $26 \mathrm{~m}$ antenna operated by Hartebeesthoek RAO, to obtain coverage of some sources over longer periods of time. In this paper we focus on the ATCA program, which provides the majority of the data for this project. The ATCA provides full polarisation information, so we are monitoring the IDV behaviour of Stokes I, Q, U, and V simultaneously at 4.8 and $8.6 \mathrm{GHz}$. These frequencies were chosen since they have low levels of confusion, are relatively free of interference, and for most sources, lie close to the transition frequency between weak and strong scattering, where strong IDV is seen (Walker 1998).

We observe each source for several minutes approximately every hour, for as long as the source is up each day. Clearly, if $t_{\text {char }}$ is $\sim 1 \mathrm{~h}$ or less, it is necessary to sample more frequently. This has been the case for one source, PKS 1257-326. Because this source shows such rapid IDV, we have observed it in several additional, shorter, more intense observing sessions.

\section{Calibration and Data Analysis}

Analysis of data for this project is ongoing. Here we report on our basic strategy and findings so far. Because we include several non-IDV sources in our program, we are able to calibrate for effects of antenna gain amplitude variation, mostly a function of elevation and time. Online system temperature corrections at the ATCA mean that errors in the visibility amplitudes from the correlator are small, except in extremely bad weather where the signal may be attenuated. We have found that at $8.6 \mathrm{GHz}$, antenna gains vary by $2-3 \%$ as a function of elevation. At $4.8 \mathrm{GHz}$, the gain-elevation effect is smaller. We also see slow amplitude variations with time, typically up to 1-2\% over the course of a day. After correcting for these effects using data on unresolved, non-IDV sources, we estimate that in fair weather, the rms error in the measurement of Stokes I (total flux density) is $<0.5 \%$. We perform phase self-calibration assuming a point source model, to correct for atmospheric phase variations. We also solve for antenna polarisation leakage terms, which we find do not vary significantly over the 2-day duration of an observing session. This correction gives accurate measurements of Stokes Q, U, and V.

The ATCA is a linear east-west array, thus the response to source structure and confusing sources within the beam varies over the course of a day as the Earth rotates. Several sources in our sample are slightly affected by confusing sources in the beam at 4.8 and $8.6 \mathrm{GHz}$, or have up to a

Table 1. IDV sources in the ATCA sample

\begin{tabular}{lccccc}
\hline Name & ID & Redshift & Optical magnitude & $\left\langle S_{4.8}\right\rangle(\mathrm{Jy})$ & $\alpha_{r}\left(S_{\nu} \propto \nu^{\alpha}\right)$ \\
\hline AO 0235+164 & BL Lac & 0.940 & 19.0 & 1.5 & 0.2 \\
PKS 0405-385 & Quasar & 1.285 & 18.0 & 0.9 & 0.3 \\
PKS 0537-441 & BL Lac & 0.894 & 15.5 & 4.6 & 0.2 \\
PKS 1034-293 & BL Lac & 0.312 & 16.5 & 1.5 & 0.3 \\
PMN J1047-6217 & - & - & 18.9 & 0.7 & 0.5 \\
PKS 1144-379 & BL Lac & 1.048 & 16.2 & 1.3 & 0.5 \\
PKS 1257-326 & Quasar & 1.256 & 18.7 & 0.22 & 0.06 \\
PMN J1326-5256 & - & - & 20.4 & 1.5 & 0.2 \\
PKS 1415-349 & - & - & 18.5 & 0.5 & -0.2 \\
PKS 1519-273 & BL Lac & - & 18.5 & 1.7 & 0.2 \\
PKS 1622-253 & Quasar & 0.786 & 21.9 & 2.3 & 0.7 \\
PKS 1622-297 & Quasar & 0.815 & 20.5 & 0.3 & -0.06 \\
PKS 2155-304 & BL Lac & 0.116 & 14.0 & &
\end{tabular}

Note: Values for optical magnitude, $4.8 \mathrm{GHz}$ flux density, and radio spectral index are indicative only. Most sources are highly variable. 
few per cent of their total flux density in an extended component which is resolved by the ATCA. In these cases, the fields are imaged so that effects of background confusing sources or extended structure can be subtracted from the visibilities. For all other sources, the IDV parameters are the same on all baselines and hence are calculated directly from the visibilities.

\subsection{IDV Parameters}

The modulation index, $\mu$, defined as the rms fractional variability $\left(\mu=\sigma_{S} / \bar{S}\right)$, is used as a measure of the level of IDV in a source. As a first estimate of the timescale of variability, the number of maxima and minima in the light curve are counted by eye, and the timescale is defined as the mean time between a minimum and the next maximum, or a maximum and the next minimum. More formally, we calculate the structure function, defined as

$$
\Sigma(\tau)=\left\langle(S(t)-S(t+\tau))^{2}\right\rangle
$$

where $S(t)$ is the measured flux density of a source at time $t$ (e.g. Spangler et al. 1989). As our sampling intervals are generally not uniform, we bin the structure function into time lag intervals. Detailed structure function analysis of the data is underway. As scintillation is a stochastic process, in measuring $t_{\text {char }}$ we are limited by the number of maxima and minima observed in the light curve. For sources with $t_{\text {char }} \sim 12 \mathrm{~h}$ or longer, there is a significant error associated with the measurement of $t_{\text {char }}$ from only two days of data. Unfortunately, only a few sources have $t_{\text {char }}<12 \mathrm{~h}$. We expect the single dish observations to improve the measurement of $t_{\text {char }}$ in sources which show large amplitude variability over somewhat longer timescales, by extending the light curves over several days, and in the case of the telescope at Hartebeesthoek $\left(122^{\circ}\right.$ west of the ATCA), filling in gaps in the data. Also, as $t_{\text {char }}$ is sometimes shorter for Stokes Q and $\mathrm{U}$ than for Stokes I, we may be able to measure $t_{\text {char }}$ with greater accuracy in polarised flux density. The polarisation parameters will assist with modelling source structure and determining ISM velocity from the annual cycle.

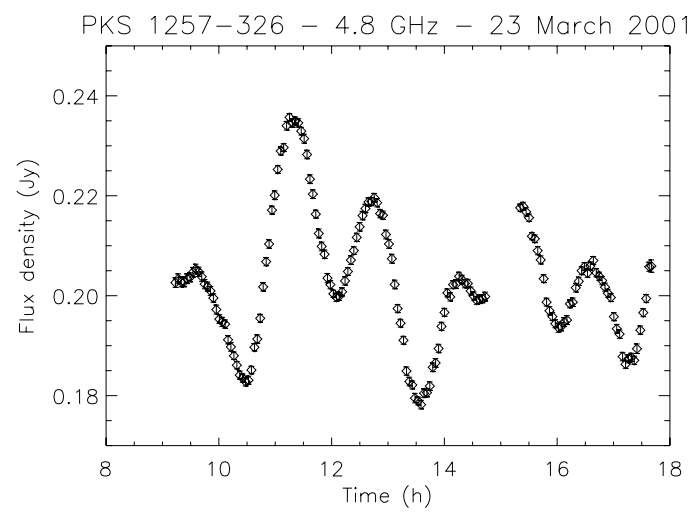

\section{Results for Individual Sources}

We present here preliminary results for three sources from the first four months of this program. These are the first IDV data to be presented for these sources, as IDV in each of them has been discovered only recently. For two of these sources, PMN J1047-6217 and PMN J1326-5256, we do not yet have enough data to tell whether or not an annual cycle in $t_{\text {char }}$ is likely to be present, while for PKS 1257-326 earlier data are available and results are suggestive of an annual cycle being present in the IDV behaviour.

\subsection{PKS 1257-326}

IDV in PKS 1257-326 was discovered serendipitously in archival ATCA data from 1995 which we re-examined in 2000. The source was re-observed in June 2000, confirming IDV with modulation index $\mu \sim 0.09$ and a timescale of $\sim 2.5 \mathrm{~h}$. This source was identified in the Deep X-ray Radio Blazar Survey (Perlman et al. 1998) as a quasar at a redshift of 1.256. In February 2001, this source was found to be varying with $t_{\text {char }}<1 \mathrm{~h}$. PKS $1257-326$ has an average linearly polarised flux density $\bar{p} \approx 6 \mathrm{mJy}, \sim 3 \%$ of the total intensity. IDV is also observed in $p$, although as this source is the weakest in our sample, we are limited by $\mathrm{S} / \mathrm{N}$ and not sensitive to small fluctuations in $p$. We have seen no evidence for dramatic variability in this source on long timescales; the mean flux density and spectrum have remained relatively stable. This may indicate that intrinsic changes in this source occur only on very long timescales, making this source a good candidate for detection of an annual cycle.

Figure 1 shows the ATCA light curve from 23 March 2001 , as well as a plot of $t_{\text {char }}$ vs fraction of year. The points on the right-hand side of the dotted line in this plot are from sparse data taken in 2000, hence the error bars associated with these are larger than those for 2001 data. The characteristic time, $t_{\text {char }}$, was measured from structure functions constructed for each epoch of data. In all cases $\Sigma(\tau)$ rises sharply to some point, then oscillates about the saturation value. The lag corresponding to the

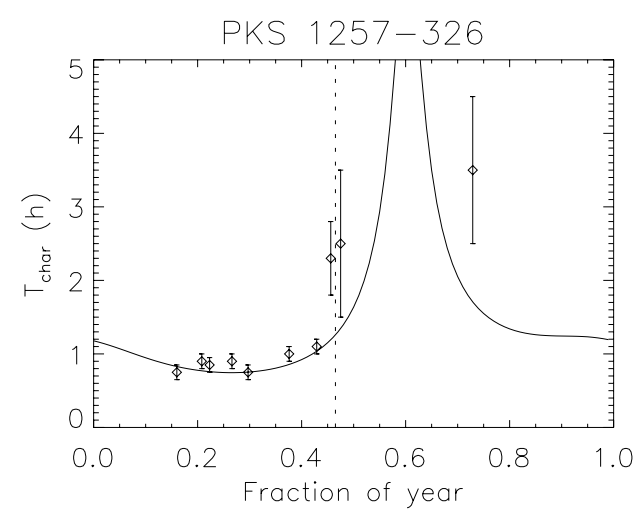

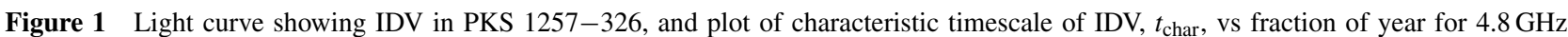
data collected so far on this source. Points to the right of the dotted line are from data taken in 2000; points left of the dotted line are data taken in 2001. The curved solid line shows one possible annual cycle which might be expected from the change in the Earth/ISM velocity alone (see text). 

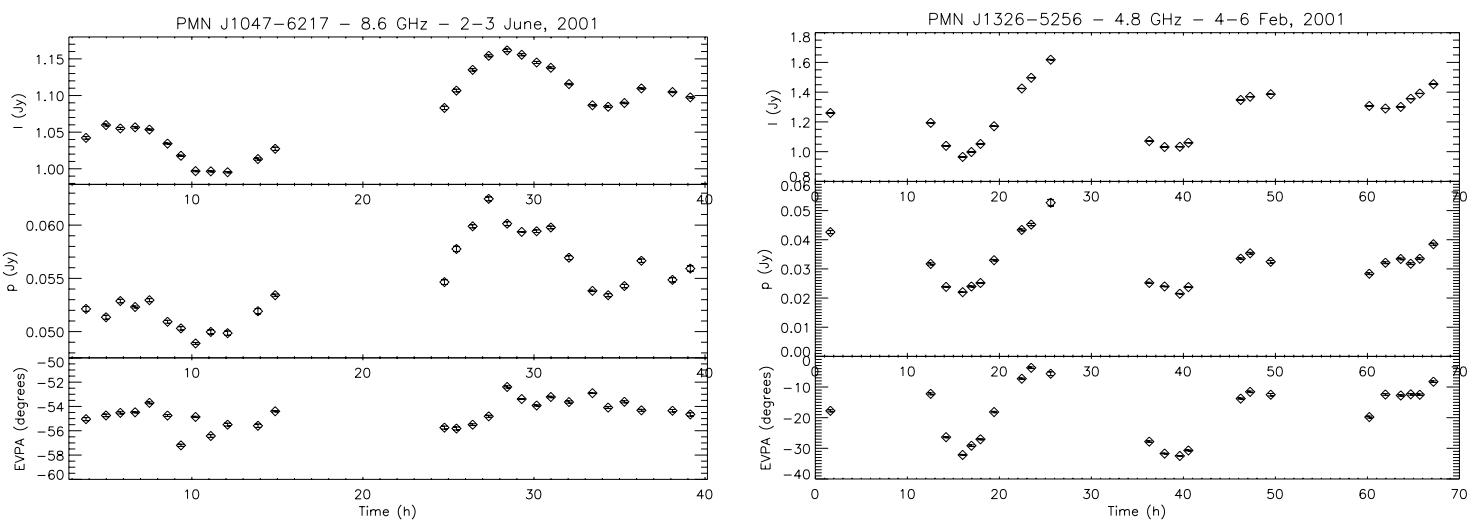

Figure 2 Light curves showing IDV in total flux density (I), linearly polarised flux density (p), and polarisation position angle for PMN J1047-6217 and PMN J1326-5256.

first maximum of $\Sigma(\tau)$ was estimated by eye, and $t_{\text {char }}$ was defined to be this value. The size of the lag bins used varied somewhat according to sampling. As a preliminary error estimate, the range of values over which the actual peak of $\Sigma(\tau)$ could lie was estimated. For the final analysis, we intend to use Monte Carlo simulations to estimate errors associated with calculating $t_{\text {char }}$ in this way from a finite sample of a stochastic variation.

The solid line shown in the plot of $t_{\text {char }}$ is the annual variation which may be expected for a scattering screen moving with the Local Standard of Rest (B. J. Rickett 2001, private communication). The free parameter here is the scintillation length scale, dependent on ISM properties. This has been set so that the curve passes through the data points for minimum $t_{\text {char }}$. This is not a formal fit, and is intended only to give an idea of what may be expected. Figure 1 suggests that an annual cycle may be present in this source, and if so, there is some indication that the 'slow-down' period is broader than expected from a change in $\mathbf{v}_{\text {ISS }}$ alone. Anisotropy in the source or the ISM could be responsible for such broadening. With further observations over the next few months, we will be able to confirm whether or not an annual cycle is present in the IDV of PKS 1257-326.

\subsection{PMN J1047-6217}

This source is one of the phase calibrators in the ATCA database. Frequent observations since 1992 showed that it is a long-term variable (S. White \& R. Duncan 2001, private communication), however IDV was not confirmed until 2001. Examination of the archival data showed little evidence for IDV in earlier data, compared with the 2001 data which show IDV at $8.6 \mathrm{GHz}$ with $\mu \sim 0.03-0.05$. If the IDV in this source is transient, it may be difficult to observe an annual cycle even if one is present. The IDV is much stronger at $8.6 \mathrm{GHz}$ than at $4.8 \mathrm{GHz}$ in this source. In fact, virtually no IDV is detected at $4.8 \mathrm{GHz}$, although it is highly variable on longer timescales. This is not unexpected from scintillation theory for a source at such low Galactic latitude, $b=-2.8^{\circ}$, as the transition frequency between weak and strong scattering increases close to the Galactic plane (Walker 1998). Thus $4.8 \mathrm{GHz}$ is likely to be well below the transition frequency, in the regime of strong (refractive) scattering, where the characteristic timescale is longer and the modulations smaller than at frequencies closer to the transition. Figure 2 (left) shows the IDV in total flux density, linearly polarised flux density, and polarisation position angle. A clear correlation can be seen here between IDV in total flux density and linear polarisation.

\subsection{PMN J1326-5256}

This source was observed during a search for calibrators suitable for $22 \mathrm{GHz}$ observations with the upgraded ATCA. It was found to be rapidly and strongly variable with an inverted spectrum (R. J. Sault 2001, private communication). Observations in early 2001 showed IDV at 4.8 and $8.6 \mathrm{GHz}$. Figure 2 (right) reveals a strong correlation in the IDV in total flux density and polarised flux density in this source at $4.8 \mathrm{GHz}$ in February 2001. At other epochs the correlation is not always so clear, suggesting that there may be more complex polarised structure in this source.

\section{Summary}

In order to understand radio variability in compact AGN it is important to understand how ISS may affect the observed variability. Quasars which show rapid variability must have very small angular sizes and therefore are affected by ISS. Recent evidence (e.g. Dennett-Thorpe \& de Bruyn 2001; Jauncey \& Macquart 2001; Rickett et al. 2001) establishes ISS as the principal mechanism responsible for IDV at $\mathrm{cm}$ wavelengths in at least some extragalactic sources. Scintillation can be used as a probe of both the ISM and source structure. The orbital motion of the Earth can produce an annual cycle in the velocity of the observed scintillation pattern. This in turn affects the characteristic timescale of the observed variability. We are undertaking an observational study to look for an annual cycle in the IDV behaviour of a number of sources. If an annual cycle is observed, we will be able to deduce the velocity of the ISM, as well as model source/ISM structure on scales of $\sim 10 \mu$ as. Many flat-spectrum radio sources have structures which are unresolved even on 
VLBI baselines to space. ISS probes smaller-scale structures, and therefore may give better estimates of brightness temperature, which is critical to understanding emission mechanisms in these objects.

\section{Acknowledgments}

The Australia Telescope Compact Array is part of the Australia Telescope which is funded by the Commonwealth of Australia for operation as a National Facility managed by CSIRO.

\section{References}

Dennett-Thorpe, J., \& de Bruyn, A. G. 2001, in IAU Symp. 205, Galaxies and their Constituents at the Highest Angular Resolutions, eds R. T. Schilizzi, S. N. Vogel, F. Paresce, \& M. S. Elvis (San Francisco: ASP), 68
Fuhrmann, L., et al. 2001, PASA, 19,

Heeschen, D. S. 1984, AJ, 89, 1111

Heeschen, D. S., Krichbaum, T., Schalinski, C. J., \& Witzel, A. 1987, AJ, 94, 1493

Kedziora-Chudczer, L. L., Jauncey, D. L., Wieringa, M. H., Tzioumis, A. K., \& Reynolds, J. E. 2001, MNRAS, 325, 1411

Kraus, A., Witzel, A., Krichbaum, T. P., Lobanov, A., Peng, B., \& Ros, E. 1999, A\&A, 352, L107

Jauncey, D. L., \& Macquart, J.-P. 2001, A\&A, 370, L9

Lovell, J. E. J. 1997, PhD Thesis, University of Tasmania, Australia Perlman, E. S., et al. 1998, AJ, 115, 1253

Quirrenbach, A., et al. 1992, A\&A, 258, 279

Rickett, B. J., Witzel, A., Kraus, A., Krichbaum, T. P., \& Qian, S. J. 2001, ApJ, 550, L11

Romero, G. E., Combi, J. A., \& Colomb, F. R. 1994, A\&A, 288, 731

Spangler, S., Fanti, R., Gregorini, L., \& Padrielli, L. 1989, A\&A, 209, 315

Walker, M. A. 1998, MNRAS, 294, 307 